\title{
Optical control over transmission of terahertz radiation through arrays of subwavelength holes of varying size
}

\author{
T. H. Isaac, ${ }^{1}$ J. Gómez Rivas, ${ }^{2}$ and E. Hendry ${ }^{1}$ \\ ${ }^{1}$ School of Physics, University of Exeter, Stocker Road, Exeter EX4 4QL, United Kingdom \\ ${ }^{2}$ FOM Institute for Atomic and Molecular Physics AMOLF, c/o Philips Research Laboratories, High Tech Campus 4, \\ 5656 AE Eindhoven, The Netherlands
}

(Received 24 September 2009; revised manuscript received 27 October 2009; published 25 November 2009)

\begin{abstract}
We modulate the transmission of terahertz $(\mathrm{THz})$ radiation through periodic arrays of subwavelength holes in a metallic film by using pulses of visible-wavelength light to photoexcite the semiconducting substrate of the hole arrays. By varying the photodoping level of the semiconductor we are able to switch off the resonant transmission of $\mathrm{THz}$ radiation through the array. By varying the size of the holes, we demonstrate the crucial role that surface modes play in the resonant transmission and ultimately in the photomodulation behavior of these structures. We demonstrate that the surface-wave transmission mechanism can allow for very efficient optical modulation of radiation transmission.
\end{abstract}

DOI: $10.1103 /$ PhysRevB.80.193412

PACS number(s): 71.45.Gm, 41.20.Jb, 84.40.-x

Photonic structures which incorporate some degree of dynamic control over their electromagnetic properties ${ }^{1,2}$ are interesting for numerous reasons. Some structures have direct applications in proposed photonic devices; in others the dynamic control can provide direct evidence for transmission pathways and for the role of material properties in determining the behavior of a structure. ${ }^{3}$ Manipulating material properties optically $^{4-10}$ is of particular interest, as changes to the structure can be made on the same time scale as the transit of light pulses through the system.

One very fundamental photonic structure is an array of subwavelength holes perforated in a conducting screen. Such arrays can exhibit narrow transmission resonances ${ }^{11}$ for wavelengths determined by the periodicity of the array-this is known as extraordinary optical transmission or EOT. The mechanisms underlying EOT in these arrays have been the subject of considerable debate, ${ }^{12-14}$ however consensus has gradually emerged that for many structures the transmission is mediated at least in part by electromagnetic surface modes at the interface between the perforated conducting screen and the dielectric layers by which it is bound. ${ }^{15,16}$

In this contribution we use pulses of visible light to modulate the transmission of terahertz $(\mathrm{THz})$ radiation through periodic arrays of subwavelength holes in a metallic film fabricated at the interface of a substrate of crystalline silicon. By varying the photodoping level of the silicon we are able to switch off EOT of $\mathrm{THz}$ radiation through the array. By varying the size of the holes we are able to explain the photomodulation effects in terms of the properties of the surface mode which mediates the enhanced transmission; in particular, we can make a direct link between the lifetime of the surface mode and the magnitude of the photomodulation. We show that if we extend the surface-mode lifetime by minimizing losses and reducing the hole size it is possible to attain photomodulation levels which are orders of magnitude greater than those found for a plain silicon surface.

The hole-array structure we shall consider in this work (Fig. 1) is formed from a 150-nm-thick film of gold on a silicon substrate. The gold is perforated with a square lattice (pitch $100 \mu \mathrm{m}$ ) of square subwavelength holes-the holes have sides ranging in size from 25 to $85 \mu \mathrm{m}$. Previous works have shown ${ }^{6,17-19}$ that such hole-array structures exhibit EOT at terahertz frequencies. The mechanism underlying the transmission can be considered as a Fano-type picture in which resonances arise from constructive interference between radiation which has been transmitted straight through the holes, and radiation which has been transmitted after coupling to a surface mode on the metal-dielectric interface. ${ }^{16}$ The frequencies of the EOT resonances $\nu_{r}$ are determined by the hole-array lattice pitch $d$ and the permittivity of the dielectric substrate, $\varepsilon_{d}$ and are approximately given by

$$
\nu_{r}=\frac{\omega_{r}}{2 \pi} \approx \frac{c \sqrt{i^{2}+j^{2}}}{d \sqrt{\varepsilon_{d}}},
$$

where $i$ and $j$ are integers indicating the diffracted order coupling to the surface mode. We determine that the hole arrays under investigation have two EOT resonances in the measured frequency range of $0.4-1.4 \mathrm{THz}$, labeled as the $\langle 1,0\rangle$ and $\langle 1,1\rangle$. Both are due to surface modes on the interface between the silicon substrate and the gold film (modes on the opposing gold-air interface lie outside the spectral range of the incident terahertz pulse, above $3 \mathrm{THz}$ ). In this work we chiefly discuss the lowest-frequency $(\langle 1,0\rangle)$ resonance as changes to the relative coupling intensity between the higher-order modes are complex and highly dependent on sample geometry. ${ }^{19}$ The amplitudes and widths of the transmission resonances are determined by the lifetime of the surface mode mediating the transmission. ${ }^{20}$ For a hole array made from lossless materials the lifetime of the surface mode is entirely determined by phase retardation across the width of the holes; i.e., arrays with larger holes exhibit a shorter mode lifetime. ${ }^{19,20}$ Through photoexcitation of the silicon surface in these samples one can effectively control this surface-mode-mediated element of the EOT transmission and alter the amplitude of the EOT resonances. ${ }^{6}$

In Fig. 1(a) we show a schematic of the experimental measurement. Two pulses (both arriving at a repetition rate of $1.05 \mathrm{kHz}$ ) separated by a 40 ps optical delay illuminate 


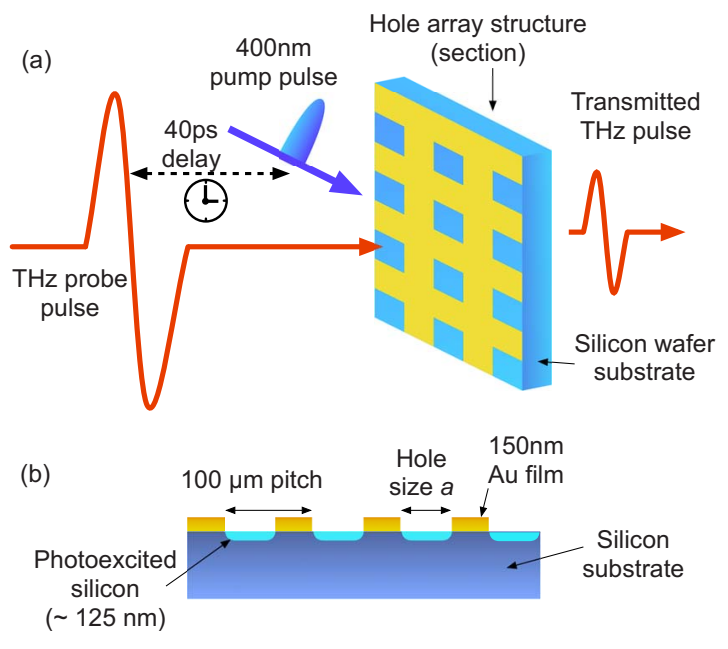

FIG. 1. (Color online) (a) Diagram of the experimental measurement. A visible pump pulse with center wavelength $400 \mathrm{~nm}$ illuminates an array of holes in a gold film on substrate of silicon. $40 \mathrm{ps}$ after the arrival of the pump pulse, a probe pulse in the $\mathrm{THz}$ range is transmitted through the hole array and subsequently detected in the far field. (b) Side profile of the photoexcited structure, indicating the $100 \mu \mathrm{m}$ array pitch and hole size $a$.

the hole-array structure at the air-gold interface. The first pulse to arrive is a $100 \mathrm{fs}, 400 \mathrm{~nm}$ pump pulse, which photoexcites the silicon exposed in the holes [Fig. 1(b)]. The 400 $\mathrm{nm}$ beam is expanded to a circle of $3 \mathrm{~cm}$ diameter to ensure that the region of photoexcitation is homogeneous across the $2 \mathrm{~cm}$ square-sided sample area. The region of silicon photoexcited is very thin, as the penetration depth of the $400 \mathrm{~nm}$ light in the silicon is submicron. ${ }^{21}$ The relaxation time for photoexcited charge carriers in silicon at room temperature is on the order of microseconds ${ }^{22}$ and so a 40 ps interval after the arrival of the pump pulse the silicon is in a steady, fully photoexcited state, i.e., we have essentially formed a thin film of extra charge carriers on the silicon interface underneath the holes. By fitting a Drude response to the measured permittivity of these carriers, ${ }^{23}$ we determine that our most intense photoexcitation beam produces a charge density in the silicon of approximately $2.8 \times 10^{24} \mathrm{~m}^{-1}$, corresponding to a plasma frequency of $30 \mathrm{THz}$. After the $40 \mathrm{ps}$ interval the $\mathrm{THz}$ probe pulse arrives in a $1 \mathrm{~cm}$ diameter collimated beam. We detect the terahertz pulse which has transmitted through the sample in the far field using a terahertz spectrometer similar to the one described in Ref. 24. In this technique we measure the electric fields of the transmitted terahertz pulses as time-domain spectra; ${ }^{19}$ these can be converted to transmission intensity spectra by taking the Fourier transform of the time-domain pulses, squaring the field amplitude, and normalizing by a reference spectrum.

In Fig. 2 we show transmission spectra taken through arrays of $45 \mu \mathrm{m}$ holes for varying fluence of the $400 \mathrm{~nm}$ pump pulses. The two expected resonances $(\langle 1,0\rangle$ and $\langle 1,1\rangle)$ are labeled. As we add an increasing fluence of $400 \mathrm{~nm}$ pulses we observe a distinct quenching of transmitted terahertz intensity at resonance. The quenching effect at the resonance peak is much higher than the quenching of the nonresonant transmission (which is dominant at lower frequencies). At

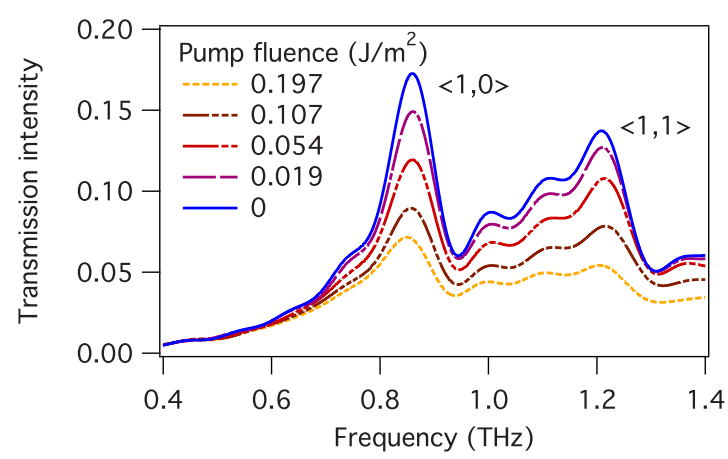

FIG. 2. (Color online) THz transmission spectra through arrays of $45 \mu \mathrm{m}$ holes with various fluences of $400 \mathrm{~nm}$ pump beam. Photoexcitation quenches the EOT resonances, indicated as the $\langle 1,0\rangle$ and $\langle 1,1\rangle$ peaks.

the $\langle 1,0\rangle$ resonance peak a pump fluence of $0.054 \mathrm{~J} / \mathrm{m}^{2} \mathrm{re}-$ duces the $\mathrm{THz}$ intensity by a factor of 0.69 whereas at 0.6 $\mathrm{THz}$, just below the resonance frequency, transmission intensity reduces by a factor of only 0.88 . In order to evaluate this enhancement in photomodulation, we can define a photomodulation ratio, $P$, as $P=\left(I_{0}-I_{p m}\right) / I_{0}$, where $I_{0}$ is the peak $\mathrm{THz}$ transmission intensity with no photoexcitation and $I_{p m}$ is the peak $\mathrm{THz}$ transmission intensity after $400 \mathrm{~nm}$ photoexcitation.

We compare these photomodulation levels directly in Fig. 3(a). In this figure we plot the photomodulation ratio $P$ at $0.86 \mathrm{THz}$ (the peak of the $\langle 1,0\rangle$ resonance) as a function of the fluence of $400 \mathrm{~nm}$ pulses for various hole sizes. As a reference we also evaluate the photomodulation measured for a plain, unstructured silicon surface. We expect the plain silicon surface to exhibit the same amount of photomodulation as the nonresonant component of the hole-array transmission, as in both cases the transmission pathways can be described by wave vectors perpendicular to the interface. The hole-array structures will introduce surface modes to the transmission-these surface modes have wave vectors parallel to the gold silicon interface. For this reason we expect the transmission resonances to be more strongly modulated than the transmission through the plain silicon interface; this effect can be seen in Fig. 3(a) as the traces for the hole arrays all lie above that for the unstructured silicon.

In Fig. 3(b) we plot the modulation as a function of hole size for various fluences, as well as for the unstructured silicon interface. We see a marked increase in the modulation as we decrease the hole size from 85 to $45 \mu \mathrm{m}$ for all fluences, indicating that as we decrease the hole size the surface mode is predominating in the transmission. However, for the very smallest holes, the trend is reversed and the resonant transmission for the 25 and $35 \mu \mathrm{m}$ holes is clearly less strongly modulated than for the $45 \mu \mathrm{m}$ holes. In a lossless hole array, one might expect that the photomodulation effect would be greatly enhanced for arrays of very small holes, as the lifetime of the surface mode is increased. Indeed, in Fig. 3(c) we plot the results of finite-element numerical modeling ${ }^{25}$ of hole arrays on a lossless silicon substrate (blue dashed line). This simple, lossless model predicts that $P$ increases monotonically upon decreasing the hole size; example transmission spectra shown as an inset in Fig. 3(c) indicate that we 

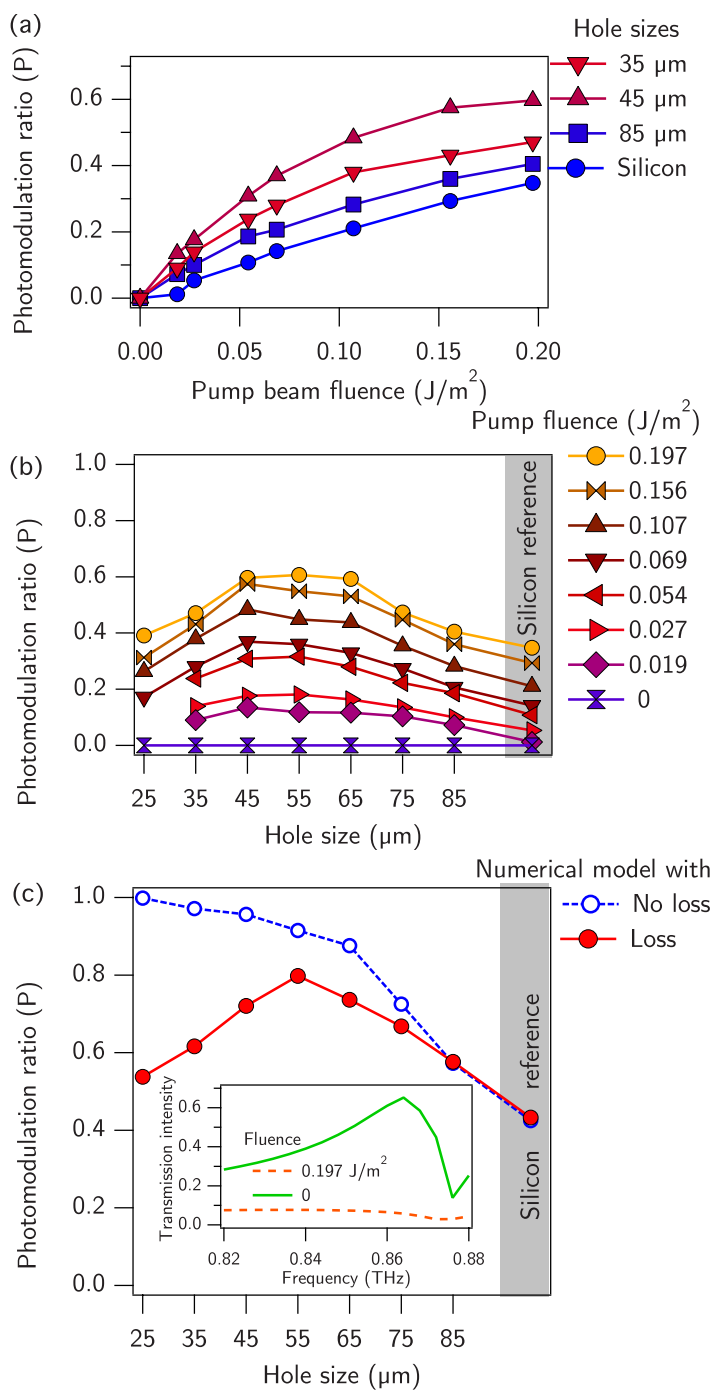

FIG. 3. (Color online) (a) Photomodulation versus fluence of $400 \mathrm{~nm}$ pump pulses for three sizes of hole and a reference surface of silicon. (b) Experimentally measured photomodulation vs hole size for various fluence of $400 \mathrm{~nm}$ pump pulses. For the $25 \mu \mathrm{m}$ holes it was not possible to measure photomodulation for the lowest fluences due to the low level of transmitted $\mathrm{THz}$ signal. (c) Numerically modeled photomodulation of the $\mathrm{THz}$ transmission at resonance as a function of hole size at a $400 \mathrm{~nm}$ pump fluence of $0.197 \mathrm{~J} / \mathrm{m}^{2}$, with and without loss in the silicon substrate. Inset: Modeled transmission spectra through $55 \mu \mathrm{m}$ holes in a lossless substrate, with and without photoexcitation.

see a similar resonance-quenching effect upon photoexcitation of the silicon substrate as we have observed in the experimental measurement. The nonmonotonic trend of photomodulation with hole size seen in our experiment is due to intrinsic losses within our silicon substrate; a low concentration of impurity charge carriers in the crystalline silicon contribute an imaginary component to the permittivity of the substrate, which reduces the surface-mode lifetime. In the 25 and $35 \mu \mathrm{m}$ holes the surface-mode lifetime is not limited by phase retardation across the holes but instead by absorption in the silicon substrate-this in turn limits the interaction of the surface modes with the photoexcited silicon. By intro- ducing an imaginary component to the dielectric constant of the silicon substrate in our model of $0.56 i$ at $0.85 \mathrm{THz}$ (determined by phase-resolved $\mathrm{THz}$ transmission measurements through the silicon substrate) we can accurately reproduce the experimental results as the solid red line in Fig. 3(c). The model incorporating loss exhibits the nonmonotonic trend in photomodulation with hole size seen in the experiment, and further demonstrates the role of surface-mode lifetime in determining the modulation. The modeled modulation for the given fluence is actually slightly higher than that measured in experiment; this is because we do not fully recover the narrow mode width for transmission through the smallest holes - the instrumental frequency resolution of the terahertz spectrometer is limited by reflections within the silicon substrate. ${ }^{19}$

Our results suggest that if one could limit the intrinsic losses in an array of very small holes fabricated on a semiconductor, the photomodulation effect could become extremely efficient. Our modeling in Fig. 3(c) indicates that for an array of $25 \mu \mathrm{m}$ holes on a lossless silicon substrate a fluence of less than $0.2 \mathrm{~J} / \mathrm{m}^{2}$ will produce a photomodulation ratio of near unity. This photomodulation ratio corresponds to the intensity $\left(I_{0} / I_{p m}\right)$ changing by a factor of 600 , compared to a factor of only 1.5 for the unstructured plain silicon surface. If we compare this to other reports of photomodulation of terahertz in literature using silicon with similar densities of photoexcited charge carriers, a silicon-based photonic crystal structure has been shown ${ }^{10}$ to modulate terahertz intensity by a factor of approximately 12 , and a siliconbased waveguide structure ${ }^{26}$ gives an intensity change of 1.4 under a continuous-wave excitation. In our lossy experimental system, the maximum intensity change attained (with the $55 \mu \mathrm{m}$ hole array) is a factor of 2.6, very comparable with other measured photomodulation measurements in hole-array structures. $^{4,6}$ In order to approach the modeled factor of 600 one would need to reduce the levels of scattering and loss in the system-this can be achieved by using ultrahigh-purity semiconductor substrates and cooling to cryogenic temperatures. ${ }^{19}$ Additionally, the photoexcited carrier lifetime in silicon is relatively long; for modulation by a pseudocontinuous-wave light source this long carrier lifetime is useful, as one can build up a high carrier density with a relatively weak light source. However, in pulsed applications the long lifetime imposes a maximum repetition rate of switching. This constraint could be avoided by using direct band-gap semiconductors such as GaAs instead of silicon. ${ }^{8}$

In conclusion, we have shown how photomodulation of the EOT in our hole-array structures is governed by the effects of surface-mode propagation. By adding a metal holearray structure to the surface of a silicon substrate we are able to enhance the response of the silicon to photoexcitation. In particular, we find that when surface-mode lifetime is limited (such as by intrinsic loss in the substrate) the photomodulation enhancement is similarly limited. However our modeling shows that by following the strategies for loss reduction and increasing the surface-mode lifetime extremely high efficiencies for optical modulation could be achieved using this scheme. Such efficient optical modulators could find applications as nonlinear switches in optical circuitry and related systems. 
T.H.I. and E.H. acknowledge the support of the EPSRC (U.K.); E.H. also acknowledges the RCUK (U.K.). This work is part of the research program of the Stichting voor Fundamenteel Onderzoek der Materie (FOM), which is financially supported by the Nederlandse organisatie voor Wetenschappelijk Onderzoek (NWO) and is part of an industrial partnership program between Philips and FOM.
${ }^{1}$ H.-T. Chen, W. J. Padilla, J. M. O. Zide, A. C. Gossard, A. J. Taylor, and R. D. Averitt, Nature (London) 444, 597 (2006).

${ }^{2}$ H.-T. Chen, H. Lu, A. K. Azad, R. D. Averitt, A. C. Gossard, S. A. Trugman, J. F. O'Hara, and A. J. Taylor, Opt. Express 16, 7641 (2008).

${ }^{3}$ J. Gómez Rivas, J. A. Sánchez-Gil, M. Kuttge, P. Haring Bolivar, and H. Kurz, Phys. Rev. B 74, 245324 (2006).

${ }^{4}$ H.-T. Chen, W. J. Padilla, J. M. O. Zide, S. R. Bank, A. C. Gossard, A. J. Taylor, and R. D. Averitt, Opt. Lett. 32, 162 (2007).

${ }^{5}$ C. Janke, J. Gómez Rivas, P. Haring Bolívar, and H. Kurz, Opt. Lett. 30, 2357 (2005).

${ }^{6}$ E. Hendry, M. J. Lockyear, J. Gómez Rivas, L. Kuipers, and M. Bonn, Phys. Rev. B 75, 235305 (2007).

${ }^{7}$ E. Hendry, F. J. Garcia-Vidal, L. Martin-Moreno, J. Gómez Rivas, M. Bonn, A. P. Hibbins, and M. J. Lockyear, Phys. Rev. Lett. 100, 123901 (2008).

${ }^{8}$ A. K. Azad, H.-T. Chen, S. R. Kasarla, A. J. Taylor, Z. Tian, X. Lu, W. Zhang, H. Lu, A. C. Gossard, and J. F. O’Hara, Appl. Phys. Lett. 95, 011105 (2009).

${ }^{9}$ N. Rotenberg, M. Betz, and H. M. van Driel, Opt. Lett. 33, 2137 (2008).

${ }^{10}$ W. Zhang, A. K. Azad, J. Han, J. Xu, J. Chen, and X. C. Zhang, Phys. Rev. Lett. 98, 183901 (2007).

${ }^{11}$ T. W. Ebbesen, H. J. Lezec, H. F. Ghaemi, T. Thio, and P. A. Wolff, Nature (London) 391, 667 (1998).

${ }^{12}$ L. Martín-Moreno, F. J. García-Vidal, H. J. Lezec, K. M. Pellerin, T. Thio, J. B. Pendry, and T. W. Ebbesen, Phys. Rev. Lett. 86, 1114 (2001).

${ }^{13}$ M. M. J. Treacy, Appl. Phys. Lett. 75, 606 (1999).

${ }^{14}$ Z. Fan, L. Zhan, X. Hu, and Y. Xia, Opt. Commun. 281, 5467
(2008).

${ }^{15}$ H. Liu and P. Lalanne, Nature (London) 452, 728 (2008).

${ }^{16}$ C. Genet, M. P. van Exter, and J. P. Woerdman, Opt. Commun. 225, 331 (2003).

${ }^{17}$ H. Cao and A. Nahata, Opt. Express 12, 1004 (2004).

${ }^{18}$ D. Qu, D. Grischkowsky, and W. Zhang, Opt. Lett. 29, 896 (2004).

${ }^{19}$ T. H. Isaac, W. L. Barnes, and E. Hendry, Phys. Rev. B 80, 115423 (2009).

${ }^{20}$ F. Miyamaru, Masaki Tanaka, and Masanori Hangyo, Phys. Rev. B 74, 153416 (2006).

${ }^{21}$ G. E. Jellison, Jr. and F. A. Modine, Appl. Phys. Lett. 41, 180 (1982).

${ }^{22}$ F. Shimura, T. Okui, and T. Kusama, J. Appl. Phys. 67, 7168 (1990).

${ }^{23}$ E. Hendry, M. Koeberg, J. Pijpers, and M. Bonn, Phys. Rev. B 75, 233202 (2007).

${ }^{24}$ A. Nahata, A. S. Weling, and T. F. Heinz, Appl. Phys. Lett. 69, 2321 (1996).

${ }^{25}$ We model the hole array using the Ansoft HFSS v.11 finiteelement modeling package, approximating the gold layer as perfect electrical conductor, modeling the silicon as a dielectric with complex permittivity of $\varepsilon_{s}=11.85+0.56 i$. We model the photoexcited silicon as a 125 -nm-thick region in the substrate immediately below the hole, with a dielectric function determined through phase-resolved direct transmission measurements of the photoexcited silicon. We define the modulation $P$ in the same way as for the experimental measurements, $P$ $=\left(I_{0}-I_{p m}\right) / I_{0}$ at resonance.

${ }^{26}$ D. G. Cooke and P. Uhd Jepsen, Opt. Express 16, 15123 (2008). 